\title{
Dark Room Prone Provocative Testing in Primary Angle Closure Suspects and those with Open Angles
}

\section{Authors}

David S. Friedman ${ }^{1,2,3} \mathrm{MD}, \mathrm{MPH}, \mathrm{PhD}$

Dolly S. Chang ${ }^{1} \mathrm{MD}, \mathrm{MPH}, \mathrm{PhD}$

Yuzhen Jiang ${ }^{3} \mathrm{MD}, \mathrm{PhD}$

Shengsong Huang ${ }^{3} \mathrm{MD}, \mathrm{PhD}$

Xiangbin Kong ${ }^{3} \mathrm{MD}, \mathrm{PhD}$

Beatriz Munoz ${ }^{1,2}, \mathrm{MS}$

Tin Aung ${ }^{4}$ MD, PhD

Paul J Foster ${ }^{5} \mathrm{PhD}$, FRCOphth

Mingguang $\mathrm{He}^{3} \mathrm{MD}, \mathrm{PhD}, \mathrm{MPH}$

\section{Institutions}

1. Wilmer Eye Institute, Johns Hopkins University, Baltimore, Maryland, USA

2. Department of International Health, Johns Hopkins Bloomberg School of Public Health, Baltimore, Maryland, USA

3. State Key Laboratory of Ophthalmology, Zhongshan Ophthalmic Center, Sun Yat-sen University, Guangzhou, China

4. Singapore National Eye Centre and National University of Singapore, Singapore

5. National Biomedical Research Centre for Ophthalmology, UCL Institute of Ophthalmology and Moorfields Eye Hospital, London, England

Conflict of Interest: The authors have no financial or other conflicts of interest concerning this study. 
Correspondence / Reprint request

David S. Friedman, MD, MPH, PhD

Wilmer 120

Baltimore, MD 21287

(410) 614-1342

(410) 955-6052

(410) 955-2542 (fax)

Email: david.friedman@jhu.edu

Word count:3137

Table:5

Figure:4 
Synopsis: The extent of IOP rise after a 15-minute dark room prone provocative test failed to separate open angle from primary angle closure suspects. It also did not predict the risk of worsening over time. 


\section{Abstract}

Purpose: To describe the results of dark room prone provocative testing (DRPPT) in primary angle closure suspects (PACS) and to compare the findings to controls with open angles.

Methods: 889 subjects with PACS in the ZAP Trial (a randomized controlled trial to compare prophylactic laser iridotomy to no treatment in PACS) and 89 with open angles in the Liwan Eye Study 5-year follow up study were placed in a dark room face down for 15 minutes. Intraocular pressure (IOP) was measured immediately before and after DRPPT.

Results: PACS participants were of similar age than controls (59.3 versus 60.5$)$, more often female (82.9 versus $58.4 \%$ ), and had lower IOP (14.3 versus $15.2 \mathrm{mmHg}$ ). The average IOP increase after DRPPT was $4.3 \pm 3 \mathrm{mmHg}$ in PACS and $5.2 \pm 2.8$ in controls $(p<0.05) .20 .5 \%$ of controls and $13.9 \%$ of those with PACS developed an IOP spike $\geq 8 \mathrm{mmHg}$ after DRPPT $(p<0.05)$. Among PACS, $15.8 \%$ of those with all four quadrants closed had an IOP elevation of $\geq 8 \mathrm{mmHg}$ as opposed to $10.0-12.4 \%$ with two or three closed quadrants $(p<0.05)$. DRPPT failed to predict who would reach a clinical trial endpoint over 6 years follow-up of those with PACS.

Conclusions: A modified DRPPT failed to separate PACS from those with open angle. Although the test resulted in greater IOP elevation among those PACS participants with all four quadrants closed than in those with two or three closed quadrants, it did not offer any insight into the risk of developing acute or chronic angle-closure disease over 6 years follow-up. 


\section{Introduction}

Provocative testing in glaucoma was first proposed by Grönholm in 1910, who noted an increase in intraocular pressure (IOP) in 1 of 10 patients in dark conditions, and a reduction of IOP in the light. ${ }^{1}$ The author suggested that glaucoma could be identified in some cases by assessing IOP before and after sitting in the dark for several hours. The prone test was described in 1968 by Hyams et $\mathrm{al}^{2}$, who examined 208 patients who laid face down for one hour with the forehead resting on the back of the hands (to avoid pressure on the eyes), and found a positive result (IOP rise $>/=8 \mathrm{mmHg}$ ) in $70 \%$ of 47 eyes with known angle-closure glaucoma, $49 \%$ of 35 suspected angle closure glaucoma eyes, $7 \% 56$ eyes with open angle glaucoma, and $4 \%$ of 70 normal eyes. Dark room and prone provocative testing were evaluated by Harris and Galin ${ }^{3}$ in 1972 and were both found to separate those with angle closure from those with open angles. Although it has been in use for 5 decades, few studies have assessed how well the dark room prone provocative test (DRPPT) separates those with open angles from those with angle closure or whether or not it can help distinguish which persons with angle closure are more likely to develop problems over time. While a report from China found that the number of closed angles on anterior segment OCT was predictive of an intraocular pressure (IOP) increase of $8 \mathrm{mmHg}$ or more after the DRPPT among a group of subjects with limbal anterior chamber depth $\leq 25 \%$ of corneal thickness, only one study has prospectively assessed subjects after DRPPT. In that study, the DRPPT was poorly predictive of who would develop acute or chronic angle closure. ${ }^{4}$

Much of the early research on DRPPT studied mixed populations of angle closure patients including those who had experienced an acute attack in the past as well as those with angle closure alone or more severe angle closure glaucoma. The results from those studies are difficult to apply to specific groups of patients and therefore clinicians lack guidance on whether or not to use the test and if so, how to interpret it. The present study was designed to assess how well a modified DRPPT (shortened to fifteen minutes from one hour) performed in a group of primary angle closure suspects (PACS), and compared the findings to those for persons with open angles.

\section{Methods}


Subjects with PACS were evaluated with a modified DRPPT as part of the qualification examination for the Zhongshan Angle Closure Prevention (ZAP) Trial (registered as 'ISRCTN45213099-Zhongshan Angle-closure Prevention Study' [http://www.controlledtrials.com/ISRCTN45213099]. Recruitment started in June 2008 and ended in October $2010 .^{5}$ Those with open angles were a consecutive group of subjects found to have open angles on gonioscopy who were participating in the five-year follow up of the Liwan Eye Study enrolled over a six-week period (89 of 874 total participants). The Liwan Eye Study was a population-based cohort study of persons living in the same district of Guangzhou city as those with PACS aged 50 and above. ${ }^{6}$ In brief, 11,991 residents living near the Zhongshan Ophthalmic Center (ZOC) participated in a screening evaluation and those with posterior (usually pigmented) trabecular meshwork not visible for 180 degrees or more on static gonioscopy without elevated IOP, peripheral anterior synechiae (PAS) or glaucoma were considered to have PACS and were asked to participate in the ZAP trial. These persons made up the angle closure study participants in the current analysis. The ZAP Trial was approved in China by the Ethics Committee of ZOC, and also received institutional review board approval from Johns Hopkins and Moorfields Eye Hospital (via the London School of Hygiene and Tropical Medicine). The open angle subjects in the present study were participants in the Liwan Eye Study who were being examined as part of a five-year follow up study and had open angles in all four quadrants on gonioscopy. Recruitment occurred when time was available on four separate occasions with 20 subjects recruited each time. They were enrolled under a protocol approved by the ZOC ethics board.

For the ZAP Trial, intraocular pressure at the screening examination was measured with non-contact tonometry (NCT, using a Topcon CT-80A (Tokyo, Japan)). Participants with NCT values greater than $24 \mathrm{mmHg}$ in either eye underwent Goldmann applanation tonometry (GAT) to confirm IOP elevation. Participants with GAT above $21 \mathrm{mmHg}$ were excluded.

Gonioscopy: Gonioscopy was performed on all subjects by the same glaucoma-trained ophthalmologist $(\mathrm{SH})$ who had high reliability when compared to glaucoma specialists with more than 10 years previous experience conducting angle closure research $(\mathrm{MH}$, 
weighted kappa $>0.80$ ). Following topical anesthesia, static gonioscopy was performed using a one mirror Goldmann-type gonioscopy lens (Single Mirror Gonioscope, Ocular Instruments, Bellevue, WA, USA) with low ambient illumination and a narrow, $1 \mathrm{~mm}$ long beam. Care was taken to avoid the beam falling on the pupil in order to prevent alteration of the angle configuration. If trabecular meshwork could not be seen because of marked iris convexity, slight tilting of the gonioscope was allowed to achieve an "over the hill" view by tilting the lens towards the trabecular meshwork (TM). Excessive tilting which might cause inadvertent corneal indentation was avoided. A dynamic examination was carried out by increasing the length and width of the beam, as well as increasing brightness. The examinee was asked to look directly towards the mirror of the gonioscope, bringing the adjacent rim of the gonioscope over the central cornea.

Pressure was exerted on the rim of the gonioscope in order to indent the central cornea. If iridotrabecular contact was not satisfactorily reversed, a dynamic examination with a 4-mirror gonioscope (Ocular Sussman Four Mirror Gonioscope, Ocular Instruments, Washington, USA) was carried out to determine if PAS were present. PAS were defined as acquired adhesions of the iris to the corneo-scleral wall crossing the scleral spur for a width of 1 clock hour or more resulting in tenting of the peripheral iris. Those with PAS were excluded from the trial as were those with elevated IOP as noted above. Participants with pigmented trabecular meshwork (PTM) not visible in at least two quadrants on gonioscopy (without PAS) in both eyes were diagnosed as PAC suspects (PACS) and considered eligible for the ZAP trial, and had repeat gonioscopy by a second glaucoma-trained ophthalmologist $(\mathrm{YJ})$ to confirm the findings. Those with open angles did not undergo repeat gonioscopy

\section{Ocular Biometry by A-Scan}

Axial length, anterior chamber depth and lens thickness were measured using a $10 \mathrm{MHz}$ A-mode ultrasound device (Nidek US1800) using a hard-tipped, corneal contact probe mounted on a Haag-Streit tonometer set to the intraocular pressure. The mean of 16 separate readings were recorded, together with standard deviation of each parameter. If the standard deviation for axial length measurement was $\geq 0.13 \mathrm{~mm}$ was, the reading 
was repeated up to another two times. None of the final readings had and SD $\geq 0.13$ $\mathrm{mm}$.

\section{Cataract grading}

All subjects underwent cataract grading using the Lens Opacity Classification System III (LOCS III) for nuclear color (NC), nuclear opalescence (NO), cortical (C) and posterior subcapsular cataract (PSC) at the slit lamp after pharmacologic dilation of the pupil and standard photographs were available for reference during the evaluation. ${ }^{7}$ Grading was carried out by 3 trained graders who underwent an initial period of standardization and additional training sessions by one senior glaucoma specialist over the course of the study.

\section{Limbal anterior chamber depth}

The limbal anterior chamber depth (LACD) was evaluated by a modified van Herick grading system using a slit lamp (BQ-900, Haag-Streit, Switzerland). LACD was graded clinically, with reference to standard photographs, as the depth of the temporal anterior chamber at the corneoscleral junction, expressed as a percent of the adjacent corneal thickness. ${ }^{8}$

\section{Modified Dark Room Prone Provocative Testing (DRPPT):}

A modified DRPPT was performed on both the PAC suspects and those with open angles. Following topical anesthesia and a baseline IOP measurement using a Tonopen applanation tonometer (TonoPen XL, Medtronic, Florida, USA), the subject lay facedown on a padded table in a dark room for 15 minutes with the forehead resting on a soft pillow. The subject was accompanied by a research nurse and allowed to talk so as not to fall asleep. A second IOP measurement using the same method was performed after 15 minutes. The measurement was made within 30 seconds of moving from facedown position to upright in a lighted environment.

\section{Statistical Analysis}


Data of both right and left eyes were included in the analyses. Baseline characteristics and ocular biometrics were compared between open angle and PACS groups using $t$ test with unequal variance for continuous variables, and Chi-Square tests for dichotomous variables. The association between angle closure status and IOP elevation after DRPPT was evaluated using multi-level modeling taking into account the correlation between the two eyes. Smoothed scatter plots (Lowess function) were used to examine the assumption of a linear relationship between the average Shaffer score and IOP change after DRPPT. Boxplots were used to examine the distribution of the IOP change after DRPPT by quadrants of angle closure in PACS.

Among subjects with PACS, differences between the 3 groups (defined by 2, 3, and 4 quadrants of angle closure) were analyzed using analysis of variance (ANOVA). Multilevel logistic regression analysis was performed to assess for factors associated with an IOP elevation of $5 \mathrm{mmHg}$ or $8 \mathrm{mmHg}$ or more after DRPPT. As subjects with PACS were part of a 72-month randomized controlled trial, we also assessed whether DRPPT predicted the development of trial endpoints (elevation of intraocular pressure, or peripheral anterior synechiae, or acute angle closure) over 6 years. ${ }^{5}$ All analyses were performed using Stata/SE, 13.1 (StataCorp, College Station, TX).

\section{$\underline{\text { Results }}$}

889 primary angle closure suspects (PACS) and 89 individuals from the Liwan Eye Study with open angles underwent DRPPT (all were Chinese residents of Guangzhou). PACS participants were slightly younger than open angle controls (59.3 versus 60.5 ) were more often female (82.9\% versus 58.4\%), and had lower IOP at baseline (14.3 versus 15.2, $p<0.05$ for all, Table 1, Figure 1). Furthermore, subjects with PACS had narrower angles on gonioscopy, shorter axial lengths, thicker lenses, shallower anterior chamber depths and denser cataracts $(p<0.05$ for all).

The average IOP increase after DRPPT was $4.3 \mathrm{mmHg}(\mathrm{SD}=3.0)$ in PACS and 5.2 $(S D=2.8)$ in those with open angles $(p<0.05$, Figure 2 and Table 2$) .61 .0 \%$ of those with open angles and $46.1 \%$ of those with PACS developed an IOP elevation of 5 $\mathrm{mmHg}$ or more after DRPPT ( $p<0.05$ for the difference in proportion). A similarly 
higher proportion of those with open angles developed an IOP spike of $8 \mathrm{mmHg}$ or more $(20.5 \%$ versus $13.9 \%, p<0.05)$. A threshold effect was not found in quadrants of angle closure and IOP change (Figure 3 ).

Among PACS, those with all four quadrants closed had a greater IOP increase (Figure,3-4, Table 3) than those with two or three quadrants of angle closure (Table 3). Of those with all four quadrants closed, $15.8 \%$ had an IOP elevation of $\geq 8 \mathrm{mmHg}$ as opposed to $10.0-12.4 \%$ with two or three closed quadrants $(p<0.05)$. In univariate analysis, people with all four quadrants closed had a 1.6 times higher odds of developing an IOP elevation of $8 \mathrm{mmHg}$ or more after DRPPT compared with people with 2 or 3 quadrants closed (Table 4). This association remained statistically significant after adjusting for age, gender and baseline IOP. Lower baseline IOP and narrower angle (measured by average Shaffer score) were also associated higher odds of developing an $8 \mathrm{mmHg}$ IOP elevation. Other factors such as age, gender, central ACD, axial length and lens thickness were not associated with an IOP elevation $\geq 8 \mathrm{mmHg}$. For PACS eyes that did not receive any intervention (control arm in the ZAP trial), IOP change after DRPPT was not a predictor for angle closure progression in 72 months (Table 5). 


\section{Discussion}

In this study, people with open angles were more likely to have a significant pressure rise than those with partially or fully occluded angles during a modified DRPPT. Although the test resulted in greater IOP elevation in those classified PACS in whom all four quadrants were closed compared with those with two or three closed quadrants, the results of this trial where one eye was left untreated for 72 months showed that baseline DRPPT did not predict who would go on to develop PAS, elevated IOP or an acute attack.

The DRPPT was first recommended over 40 years ago by Harris who found that those with closed angles were far more likely to have elevation in IOP than those with open angles. ${ }^{3}$ Authors at that time applied the DRPPT to a mix of persons with varying amounts of angle closure (including some who had previously had an acute attack) and assumed that an increase in IOP was a sign of a predisposition to angle closure glaucoma. The test was performed for one hour. While the DRPPT on average causes IOP elevation, it is clear from the present study that this finding is not unique to persons with angle closure.

The rationale for evaluating angle closure subjects in the dark is based on a large body of data that show that angles are more likely to close in dim illumination. Many who are open on gonioscopy in bright light will be closed when viewed in the dark. Pavlin described a dark room provocative test using UBM in eight patients who were identified as having irido-trabecular contact in response to decreased illumination. ${ }^{9} \mathrm{~A}$ high likelihood of appositional closure in the dark was also reported in a population of Japanese subjects with either suspect PAC, PAC, PACG or fellow eyes of persons undergoing an acute attack of angle closure. ${ }^{10}$ Similar findings were documented in the fellow eyes of Singapore Chinese subjects. ${ }^{11}$ Furthermore, we previously reported that the fellow eyes of persons with unilateral acute attacks have more substantial angle narrowing in the dark than normal controls. ${ }^{11}$ 
Placing persons face down theoretically should lead to anterior movement of the lens iris diaphragm. This could cause further closure of the angle and lead to an elevation of IOP. The combination of darkness and prone positioning clearly leads to IOP elevation, but elevation occurred in those with open angles as well as those with angle closure. The current study demonstrates that using the shortened DRPPT that was studied in this trial does not help separate these two groups. While previous researchers had performed DRPPT for one hour, we believed that if the test were found to have predictive value it would be much more likely to be used clinically if it were shorter. We therefore reduced the time to 15 minutes assuming that it would be adequate time to allow for movement of the lens iris diaphragm and increase in IOP. This modified DRPPT also was not useful in predicting which PACS subjects were at highest risk for progressing to primary angle closure or acute angle closure. The only prospective study that previously assessed this question followed 129 subjects at multiple centers for an average of 2.7 years. Eight patients (6\%) developed acute angle closure attacks in the follow-up period, while another 17 patients (13\%) developed primary angle closure, defined as appositional or synechial closure of 0.5 to 3 clock hours in the superior angle. ${ }^{4}$ The DRPPT was unable to accurately predict those patients who went on to angle closure. To our knowledge, the present study is the largest prospective cohort evaluating a DRPPT and again found that it failed to predict who would progress to angle closure disease or develop acute angle closure over 6 years.

Certain factors predisposed to a larger increase in IOP after the DRPPT. The finding that people with lower IOP had a larger increase in IOP after DRPPT is likely due in part to regression to the mean since the IOP fluctuates naturally and those with lower IOP would have been more likely to have a higher IOP on repeat testing and those with higher IOP would have been more likely to have a lower IOP on repeat testing.

It is unclear why those with open angles had a larger increase in IOP in response to this shortened DRPPT than those with PACS. It is possible that the trabecular meshwork is compacted with slight anterior lens diaphragm movement, even in open angles, reducing aqueous outflow. We did not measure the change in the position of the lens after prone positioning in the present study, but it is possible that this could be assessed 
with anterior segment OCT in the future. In unpublished data we previously found that there were no changes in anterior chamber depth in response to supine positioning when compared to anterior chamber depth measured when seated. Perhaps there are hydrostatic changes that occur with prone head posture since the head is no longer above the heart? This could in theory raise episcleral venous pressure and lead to increased IOP via this mechanism. Alternatively, the choroid may expand leading to a transient increase in IOP. Additional research will be required to determine the mechanism of IOP elevation and why IOP elevation was greater for those with open angles. However, if we only look at the degree of angle closure among those with PACS, we found eyes with narrower angle were more likely to develop an $8 \mathrm{mmHg} I O P$ elevation after DRPPT. It is possible that our exclusion of those with angle closure and high IOP may have limited our ability to see a difference in DRPPT across the spectrum of angle closure.

This study was community based and may not represent cases that come to the clinic. In addition, angle closure mechanisms may vary across populations and the findings from this Chinese population may not apply to others. Furthermore, we only assessed PACS subjects and the results for those with PAC or PACG may in fact be different. Finally, the DRPPT used was 15 minutes, shorter than in some previous reports, and this may have reduced the effectiveness of the test. We elected to use a 15-minute test for two reasons. First, it is clinically impractical to do the DRPPT for one hour. The long duration of the test requires full-time accompaniment to avoid the patient falling asleep, and the one-hour test is rarely used in clinical practice. Second, our previous work looking at anterior segment changes in response to illumination indicates that it these changes occur nearly instantaneously. ${ }^{12}$ We did not believe that longer prone positioning would dramatically affect the results. Additional studies are required to confirm these assumptions.

In conclusion, the DRPPT did not distinguish those PACS subjects from those with open angles in this large study of Chinese individuals. The extent of IOP rise after DRPPT also failed to predict later outcomes. 


\section{Funding:}

This work is supported by the Fight for Sight (grant no. 1655) (United Kingdom), the Sun Yat-sen University 5010 Project Fund (grant no. 2007033) (China), the National Natural Science Foundation of China (grant no. 81420108008) (China) and Fundamental Research Funds of the State Key Laboratory in Ophthalmology (China). Prof He receives support from the University of Melbourne Research at Melbourne Accelerator Program Professorship. The Centre for Eye Research Australia receives operational infrastructural support from the Victorian government. Dr. Jiang and Prof Foster supported by a grant from the British Council for Prevention of Blindness (UK). Prof Foster received additional support from the National Institute for Health Research (NIHR) Biomedical Research Centre at Moorfields Eye Hospital, London, United Kingdom (NIHR-BRC2 009; Moorfields/UCL-IOO), Special Trustees of Moorfields Eye Hospital (since renamed Moorfields Eye Charity) and the Richard Desmond Charitable Foundation (via Fight for Sight UK). These funding sources did not play any role in the design and conduct of the study; in the collection, management, analysis, or interpretation of the data; or in the preparation, review, approval of the manuscript, or decision to submit the manuscript for publication. 


\section{Reference}

1. Higgitt AC. The dark-room test. Br J Ophthalmol 1954;38:242-247.

2. Hyams SW, Friedman Z, Neumann E. Elevated intraocular pressure in the prone position. A new provocative test for angle-closure glaucoma. Am J Ophthalmol 1968;66:661-672.

3. Harris LS, Galin MA. Prone provocative testing for narrow angle glaucoma. Arch Ophthalmol Chic III 1960 1972;87:493-496.

4. Wilensky JT, Kaufman PL, Frohlichstein D, et al. Follow-up of angle-closure glaucoma suspects. Am J Ophthalmol 1993;115:338-346.

5. Jiang Y, Friedman DS, He M, et al. Design and Methodology of a Randomized Controlled Trial of Laser Iridotomy for the Prevention of Angle Closure in Southern China: The Zhongshan Angle Closure Prevention Trial. Ophthalmic Epidemiol 2010;17:321-332.

6. He M, Foster PJ, Ge J, et al. Prevalence and clinical characteristics of glaucoma in adult Chinese: a population-based study in Liwan District, Guangzhou. Invest Ophthalmol Vis Sci 2006;47:2782-2788.

7. Chylack LT, Wolfe JK, Singer DM, et al. The Lens Opacities Classification System III. Arch Ophthalmol 1993;111:831-836.

8. Van Herick W, Shaffer RN, Schwartz A. Estimation of width of angle of anterior chamber. Incidence and significance of the narrow angle. Am J Ophthalmol 1969;68:626-629.

9. Pavlin CJ, Harasiewicz K, Foster FS. An ultrasound biomicroscopic dark-room provocative test. Ophthalmic Surg 1995;26:253-255.

10. Sakuma T, Sawada A, Yamamoto T, Kitazawa Y. Appositional angle closure in eyes with narrow angles: an ultrasound biomicroscopic study. J Glaucoma 1997;6:165-169.

11. Friedman DS, Gazzard G, Foster P, et al. Ultrasonographic biomicroscopy, Scheimpflug photography, and novel provocative tests in contralateral eyes of Chinese patients initially seen with acute angle closure. Arch Ophthalmol Chic III 1960 2003;121:633-642.

12. Gus Gazzard, Paul J Foster, David S Friedman, et al. Light to dark physiological variation in irido-trabecular angle width. $\mathrm{Br} J$ Ophthalmol 2004;88:1357-1482. 
Table 1. Demographic and Biometric Characteristics of Primary Angle Closure Suspects and Open Angles

\begin{tabular}{|c|c|c|c|}
\hline & $\begin{array}{c}\text { PACS } \\
(\mathrm{N}=889)\end{array}$ & $\begin{array}{l}\text { Open Angles } \\
\qquad(\mathrm{N}=89)\end{array}$ & $p$ \\
\hline Age (yrs, mean $\pm S D$ ) & $59.30 \pm 5.01$ & $60.49 \pm 3.54$ & 0.005 \\
\hline \multirow[t]{2}{*}{ Female $[\mathrm{N}(\%)]$} & $737(82.9)$ & $52(58.4)$ & $<0.001$ \\
\hline & $\begin{array}{c}\text { PACS } \\
(n=1778)\end{array}$ & $\begin{array}{l}\text { Open Angles } \\
\qquad(n=176)\end{array}$ & \\
\hline Baseline IOP (mmHg, mean $\pm S D)$ & $14.32 \pm 2.62$ & $15.17 \pm 3.08$ & $<0.001$ \\
\hline Central ACD (mm, mean $\pm S D)$ & $2.55 \pm 0.22$ & $2.86 \pm 0.31$ & $<0.001$ \\
\hline Axial length $(\mathrm{mm} \text {, mean } \pm \mathrm{SD})^{*}$ & $22.49 \pm 0.73$ & $23.50 \pm 1.04$ & $<0.001$ \\
\hline Lens thickness (mm, mean $\pm S \mathrm{SD})^{*}$ & $4.88 \pm 0.32$ & $4.77 \pm 0.38$ & 0.011 \\
\hline \multicolumn{4}{|l|}{ Cataract grading (mean $\pm S D)^{* *}$} \\
\hline Nuclear opalescence & $2.30 \pm 0.59$ & $1.90 \pm 0.36$ & $<0.001$ \\
\hline Nuclear color & $2.18 \pm 0.57$ & $1.94 \pm 0.41$ & $<0.001$ \\
\hline Cortical & $0.84 \pm 1.16$ & $0.16 \pm 0.55$ & $<0.001$ \\
\hline Posterior subcapsular & $0.12 \pm 0.31$ & $0 \pm 0$ & $<0.001$ \\
\hline $\begin{array}{l}\text { Limbal anterior chamber depth (\% corneal } \\
\text { thickness) }\end{array}$ & $22.16 \pm 7.62$ & $53.84 \pm 26.00$ & $<0.001$ \\
\hline Average Shaffer score (mean \pm SD) ${ }^{+}$ & $1.33 \pm 0.60$ & $3.46 \pm 0.61$ & $<0.001$ \\
\hline Number of Quadrant Closed (mean $\pm S D)^{+}$ & $3.55 \pm 0.66$ & $0.15 \pm 0.41$ & $<0.001$ \\
\hline
\end{tabular}

Abbreviation: PACS, primary angle closure suspects; SD, standard deviation; N, number of persons; $n$, number of eyes.

"Measured by A-Scan with $S D \leq 0.13$

${ }^{*}$ Graded using the Lens Opacity Classification System III

${ }^{* * *}$ Graded by van Herick

+Graded by gonioscopy 
Table 2. Intraocular Pressure Change After Dark Room Prone Provocative Test by Primary Angle Closure Suspects and Open angle

\begin{tabular}{|l|c|c|c|}
\hline & $\begin{array}{c}\text { PACS } \\
(\mathrm{n}=1778)\end{array}$ & $\begin{array}{c}\text { Open Angle } \\
(\mathrm{n}=176)\end{array}$ & \\
\hline IOP change $(\mathrm{mmHg}$, mean \pm -vD $)$ & $4.25 \pm 2.99$ & $5.23 \pm 2.77$ & $<0.001^{*}$ \\
\hline IOP increase $\geq 5 \mathrm{mmHg}(\%)$ & $46.06 \%$ & $60.80 \%$ & $<0.001$ \\
\hline IOP increase $\geq 8 \mathrm{mmHg}(\%)$ & $13.89 \%$ & $20.45 \%$ & 0.018 \\
\hline$\%$ of IOP increase $(\%$, mean $\pm S D)$ & $32.1 \pm 24.6$ & $37.3 \pm 23.4$ & 0.006 \\
\hline IOP increase $\geq 20 \%$ baseline (\%) & $67.77 \%$ & $77.27 \%$ & 0.010 \\
\hline IOP increase $\geq 40 \%$ baseline (\%) & $34.93 \%$ & $40.34 \%$ & 0.152 \\
\hline IOP increase $\geq 60 \%$ baseline (\%) & $13.27 \%$ & $15.91 \%$ & 0.329 \\
\hline
\end{tabular}

Abbreviation: PACS, primary angle closure suspects; IOP, intraocular pressure; SD, standard deviation.

${ }^{*}$ Adjusted for baseline IOP 
Table 3. Intraocular Pressure Change After Dark Room Prone Provocative Test and Quadrants of Angle Closure among Primary Angle Closure Suspects

\begin{tabular}{|l|c|c|c|c|}
\hline & \multicolumn{3}{|c|}{ Quadrants of angle closure } \\
\hline & 2 & 3 & 4 & $p$ \\
& $(\mathrm{n}=162)$ & $(\mathrm{n}=481)$ & $(\mathrm{n}=1135)$ & \\
\hline IOP change $(\mathrm{mmHg}, \text { mean } \pm S D)^{*}$ & $4.23 \pm 2.85$ & $4.14 \pm 2.89$ & $4.30 \pm 3.05$ & 0.625 \\
\hline IOP change $\geq 5 \mathrm{mmHg}(\%)$ & 46.3 & 46.4 & 45.9 & 0.984 \\
\hline IOP change $\geq 8 \mathrm{mmHg}(\%)$ & 12.4 & 10.0 & 15.8 & 0.007 \\
\hline$\%$ of IOP increase (\%, mean $\pm S \mathrm{SD})^{*}$ & $33.8 \pm 26.6$ & $32.0 \pm 23.8$ & $32.0 \pm 24.6$ & 0.661 \\
\hline IOP increase $\geq 20 \%$ baseline (\%) & 69.1 & 70.1 & 66.6 & 0.368 \\
\hline IOP increase $\geq 40 \%$ baseline (\%) & 38.3 & 36.6 & 33.7 & 0.353 \\
\hline IOP increase $\geq 60 \%$ baseline (\%) & 16.7 & 10.6 & 13.9 & 0.082 \\
\hline
\end{tabular}

* One-way ANOVA, others Chi-square test. 
Table 4. Predictors for Intraocular Pressure Rise $\geq 8 \mathrm{mmHg}$ after Dark Room Prone Provocative Test in Primary Angle Closure Suspects

\begin{tabular}{|l|c|c|c|c|}
\hline & $\begin{array}{c}\text { Unadjusted Odds } \\
\text { Ratio }\end{array}$ & $p$ & $\begin{array}{c}\text { Adjusted } \\
\text { Odds Ratio* }\end{array}$ & $p$ \\
\hline Age (10 years older) & $0.93(0.71-1.21)$ & 0.583 & $0.85(0.64-1.13)$ & 0.260 \\
\hline Female & $0.81(0.57-1.13)$ & 0.218 & $0.80(0.56-1.14)$ & 0.212 \\
\hline Baseline IOP (mmHg) & $0.78(0.74-0.83)$ & $<0.001$ & $0.78(0.74-0.83)$ & $<0.001$ \\
\hline $\begin{array}{l}\text { Quadrants of angle closure } \\
4 \text { vs. 2 \& 3 }\end{array}$ & $1.58(1.18-2.13)$ & 0.002 & $1.88(1.38-2.57)$ & $<0.001$ \\
\hline Average Shaffer score & $0.65(0.52-0.81)$ & $<0.001$ & $0.58(0.46-0.72)$ & $<0.001$ \\
\hline Central ACD (mm) & $0.68(0.37-1.25)$ & 0.213 & $0.90(0.47-1.71)$ & 0.746 \\
\hline Axial Length $(\mathrm{mm})$ & $1.13(0.94-1.35)$ & 0.209 & $1.19(0.99-1.44)$ & 0.069 \\
\hline Lens thickness $(\mathrm{mm})$ & $1.14(0.75-1.74)$ & 0.544 & $1.20(0.76-1.89)$ & 0.429 \\
\hline
\end{tabular}

Abbreviation: IOP, intraocular pressure; ACD, anterior chamber depth

*Adjusted for age, gender and baseline IOP 
Table 5. The Association Between Dark Room Prone Provocative Tests and Incidence of Angle Closure in 72 Months Among Primary Angle Closure Suspects

\begin{tabular}{|l|c|l|}
\hline & Hazard ratio $(95 \% \mathrm{Cl})$ & $p$-value \\
\hline Univariate: & & \\
\hline DRPPT IOP increase $(\mathrm{mmHg})$ & $1.00(0.90-1.12)$ & 0.792 \\
\hline Multivariate: & $1.00(0.89-1.12)$ & \\
\hline DRPPT IOP increase $(\mathrm{mmHg})$ & $1.07(0.96-1.20)$ & 0.967 \\
\hline Baseline IOP $(\mathrm{mmHg})$ & $1.06(0.99-1.13)$ & 0.230 \\
\hline Age (per year increase) & $1.11(0.45-2.74)$ & 0.079 \\
\hline Female & $0.91(0.79-1.04)$ & 0.792 \\
\hline Shaffer score & & \\
\hline
\end{tabular}

${ }^{*}$ Cox proportional hazard model 
Figure 1. Distribution of Change in Intraocular Pressure after Dark Room Prone Provocative Test in Open angle and PACS

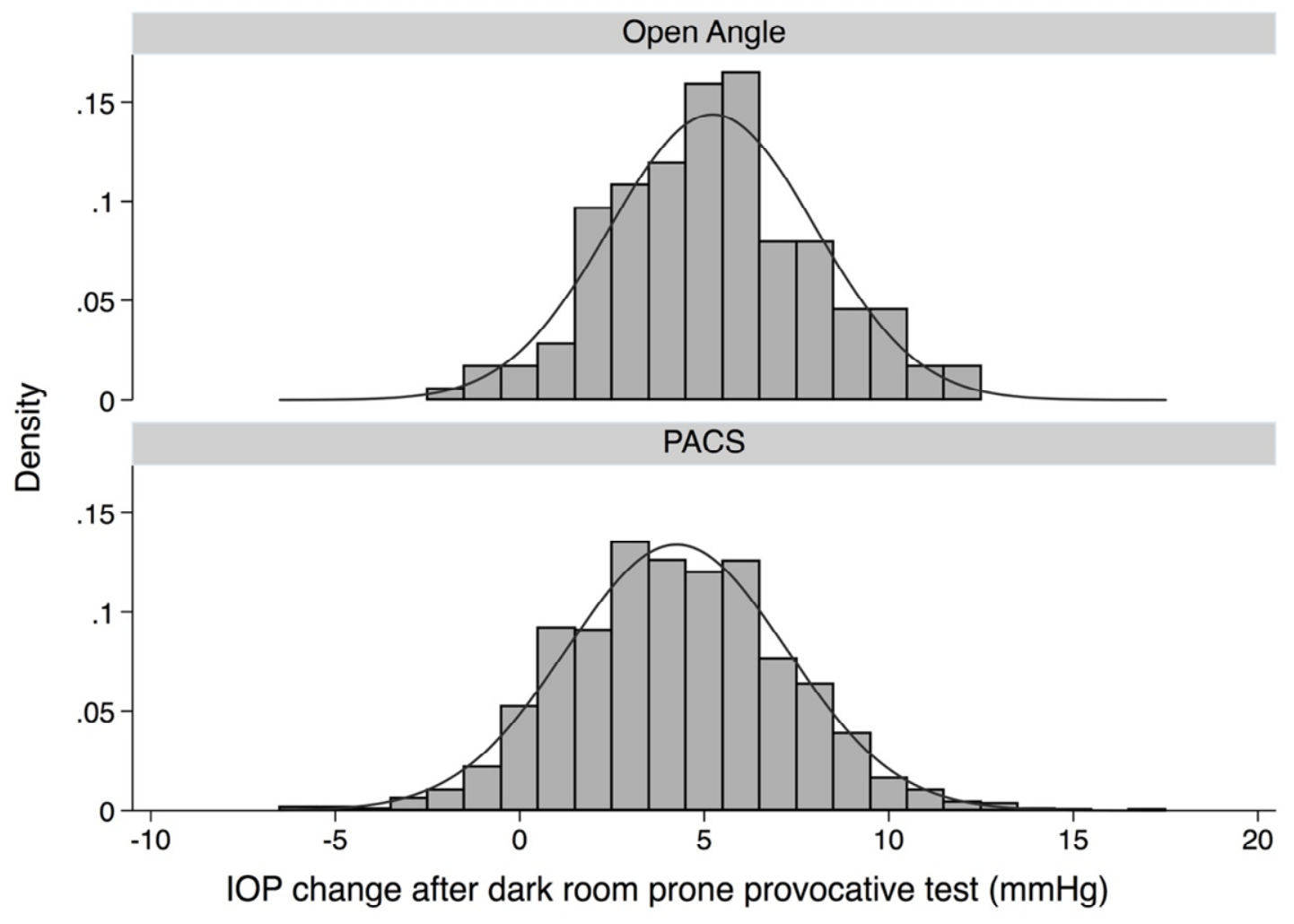


Figure 2. Intraocular Pressure Change After Dark Room Prone Provocative Test and Baseline Intraocular Pressure

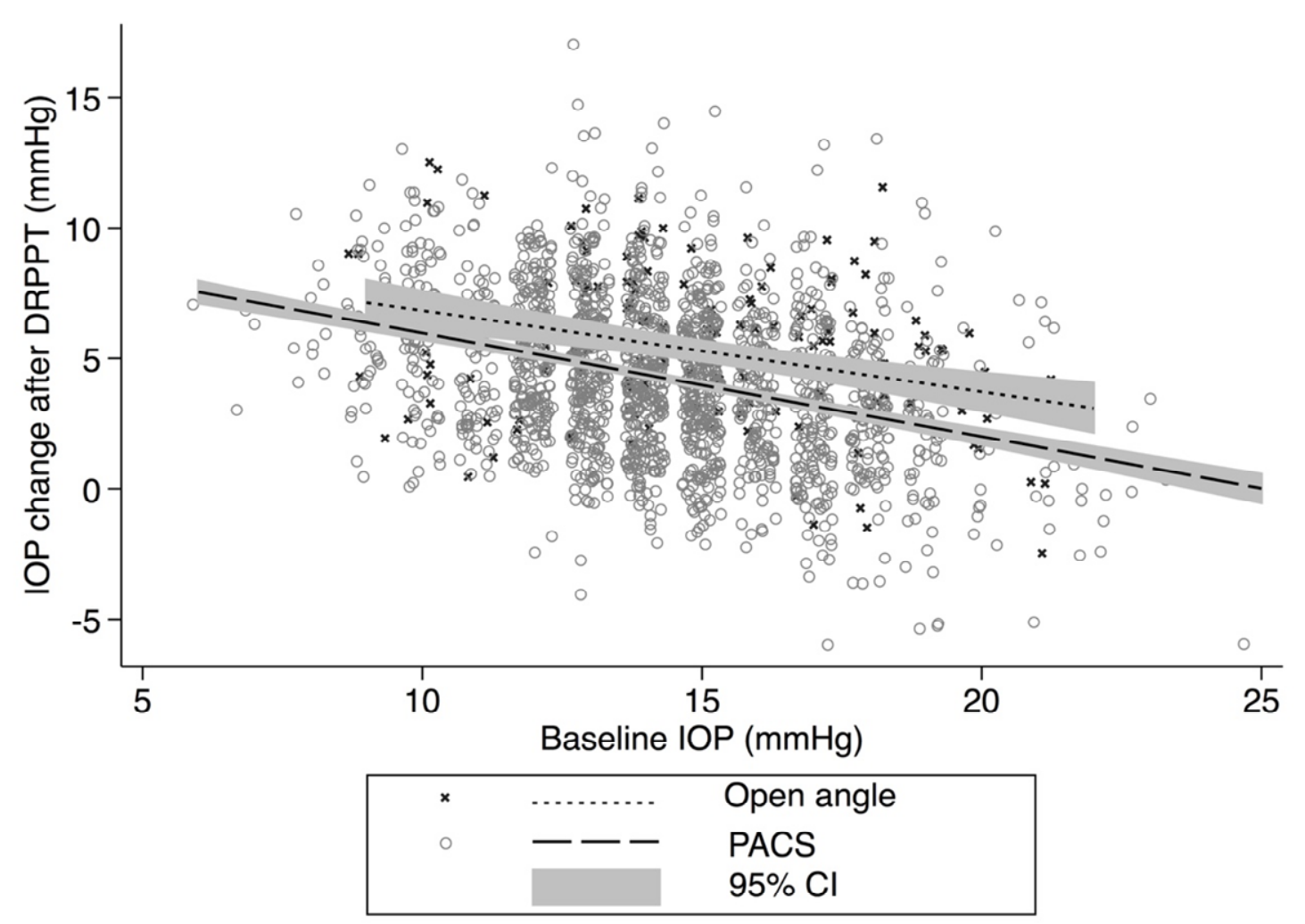


Figure 3. Distribution of Change in Intraocular Pressure after Dark Room Prone Provocative Test by Quadrants of angle closure in PACS

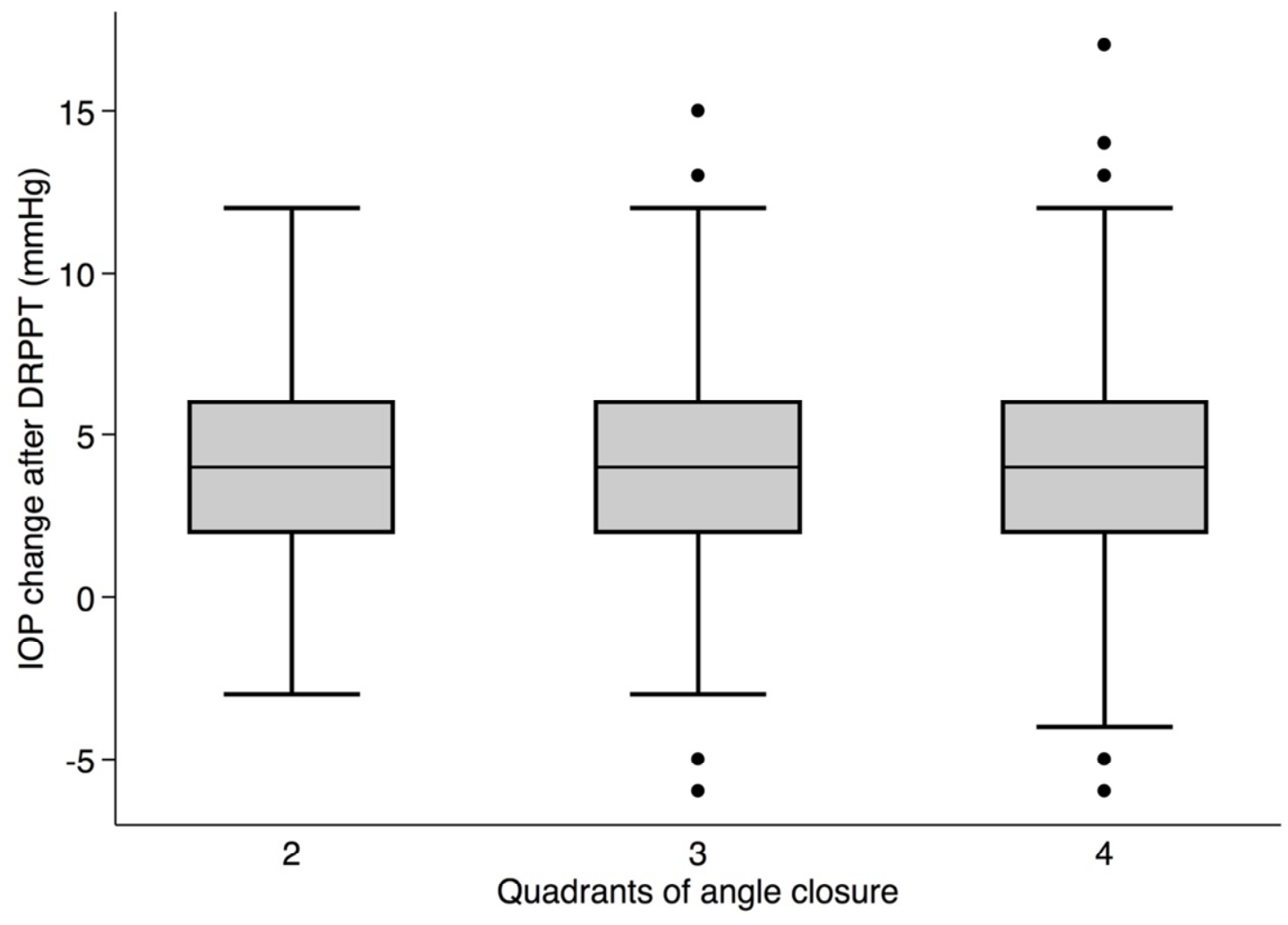


Figure 4. Lowess IOP Change after DRPPT over average Shaffer score in PACS

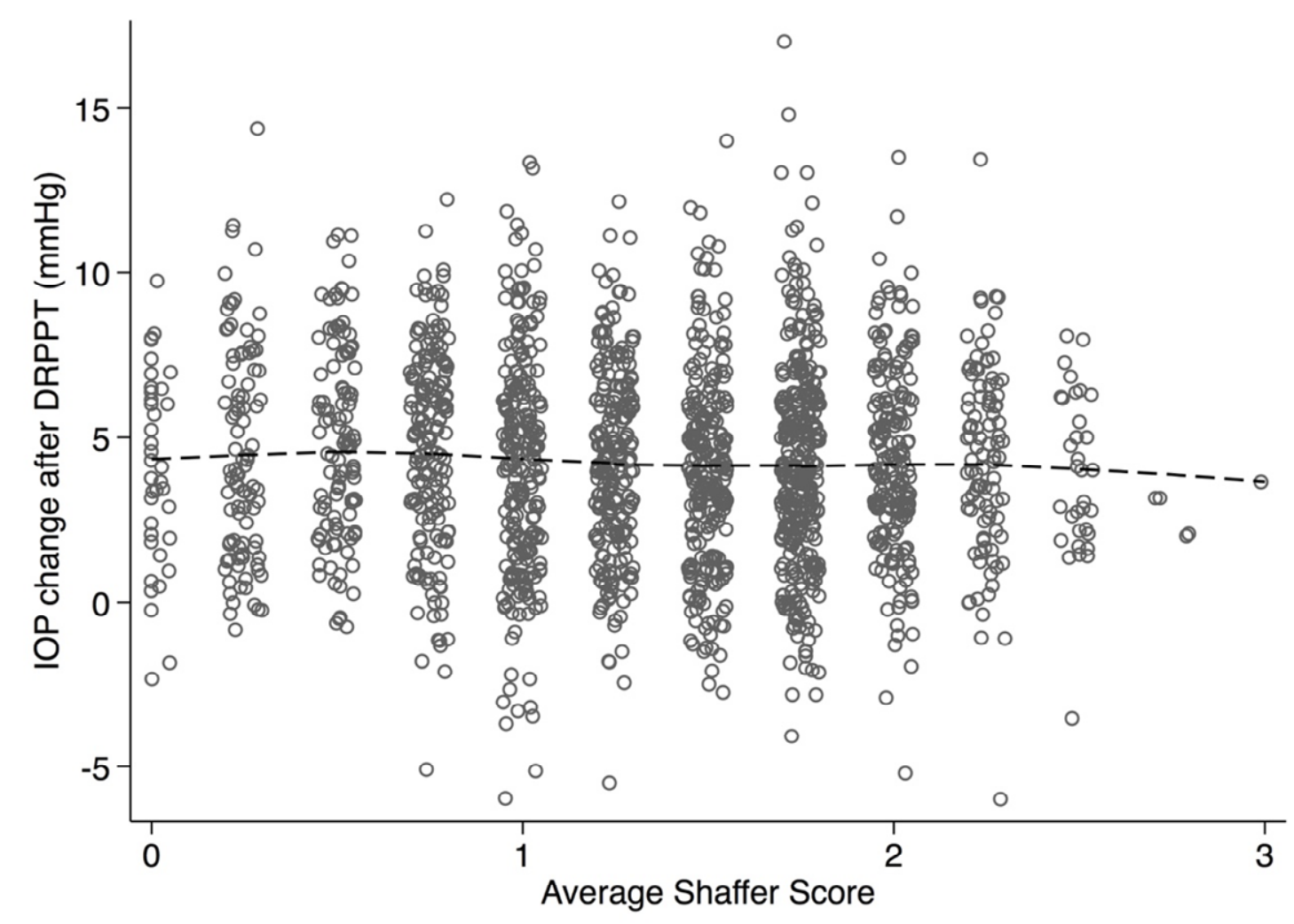

